\title{
PSPICE modeling of meminductor
}

\author{
Dalibor Biolek · Zdeněk Biolek • Viera Biolková
}

Received: 12 June 2010/Accepted: 2 July 2010/Published online: 20 July 2010

(C) The Author(s) 2010. This article is published with open access at Springerlink.com

\begin{abstract}
PSPICE models of current- and flux-controlled meminductor are described. The models consist of two parts, one of which represents the state-space description of the memory effect of the device, and the other part is an inductor whose inductance depends on the system state. The basic fingerprints of the meminductor, i.e. the fluxcurrent pinched hysteresis loops, the unambiguous constitutive relation between the time integral of flux and electric charge, and identical zero-crossing points of flux and current waveforms are demonstrated on the example of current-controlled meminductor.
\end{abstract}

\section{Keywords Meminductor $\cdot$ Model $\cdot$ SPICE}

\section{Introduction}

The discovery of solid-state memristor in Hewlett-Packard (HP) labs, reported in Nature in May 2008 [1], initiated a

D. Biolek $(\bowtie)$

Department of Microelectronics, Brno University of

Technology, Údolní 53, Brno, Czech Republic

e-mail: dalibor.biolek@unob.cz; dalibor.biolek@centrum.cz

\section{Z. Biolek}

SŠIEŘ Rožnov p.R., Školní 1610, Rožnov p.R., Czech Republic

e-mail: biolek@valachnet.cz

V. Biolková

Department of Radio Electronics, Brno University of

Technology, Purkyňova 118, Brno, Czech Republic

e-mail: biolkova@feec.vutbr.cz

D. Biolek

Department of EE, University of Defence Brno, Kounicova 65, Brno, Czech Republic growing interest in computer modeling and simulation of memristor [2-9], the fourth fundamental passive element, theoretically predicted by Chua in 1971 [10]. The reason consists in the fact that the above device is not currently available as off-the-shelf circuit. Then its model, particularly when implemented in current programs for circuit simulation such as SPICE, can serve as an important tool in computer experiments with such devices.

In addition to the memristor, other hypothetical "memdevices" have come into consideration within the last year, namely the memcapacitor and the meminductor [11-14]. As noted in [15], “...combined with the already known memristor, such elements open up new and unexplored functionalities in electronics...".

In order to enable simulation experiments with the above mem-elements, the existing PSPICE models of memristor should be complemented with similar models of memcapacitor and meminductor. The first step was performed in [16], where a general methodology for PSPICE modeling of mem-devices was introduced. The very first PSPICE model of the memcapacitor was published in [17]. To the best of our knowledge, no similar PSPICE model of the meminductor has been hitherto reported in the literature. Thereby, this Letter introduces foolproof models of current- and flux-controlled meminductors.

\section{General models of current- and flux-controlled meminductors for PSPICE}

According to [18], a network element can be defined axiomatically by its constitutive relation (CR), which does not depend on the element interaction with the surrounding networks. For the meminductor, the CR is the relation [16] 
$f(\rho, q)=0$,

where $\rho$ is the time-domain integral of magnetic flux $\varphi$ (TIF) of the meminductor, and $q$ is the electric charge, i.e. the time integral of electric current $i$ (TIC):

$\rho(t)=\int_{-\infty}^{t} \varphi(\alpha) d \alpha, \quad q(t)=\int_{-\infty}^{t} i(\alpha) d \alpha$.

If the CR (1) can be transformed such that it depicts the TIF as a single-valued function $\hat{\rho}$ of the charge, namely

$\rho=\hat{\rho}(q)$,

then we talk about a current-controlled $[12,16]$ or a charge-controlled meminductor. In terms of meminductor flux and current, CR (3) can be rewritten in the form

$\varphi=L_{M}(q) i$

where

$L_{M}(q)=\left.\frac{d \hat{\rho}(q)}{d q}\right|_{Q}$

is the small-signal meminductance defined at the operating point Q [18].

Similarly, if CR (1) can depict the charge $q$ as a singlevalued function $\hat{q}$ of the TIF,

$q=\hat{q}(\rho)$,

then (6) defines a flux-controlled $[12,16]$ or TIF-controlled meminductor. CR (6) can be transformed into the form

$i=\Lambda_{M}(\rho) \varphi$,

where

$\Lambda_{M}(\rho)=\left.\frac{d \hat{q}(\rho)}{d \rho}\right|_{Q}$ is the small-signal inverse meminductance defined at the operating point $\mathrm{Q}$.

It is shown on the example of HP memristor [1] that there is a significant difference between the behavior of the ideal hypothetical device (memristor) and its physical implementation. Since the key parameter of the device, resistance in the case of memristor, can be changed only within bounds that are given by the physical limitations of a concrete implementation, the HP memristor must be modeled as a more general memristive system [19]. Analogously, it is shown in $[12,16]$ that the memcapacitor and the meminductor are special cases of more general memcapacitive and meminductive systems. In order to model properly the meminductor also in its limit states, it should be regarded as a current- or flux-controlled meminductive system (CCMLS or FCMLS), respectively.

The CCMLS for modeling the current-controlled meminductor can be defined by the following algebraic port equation (PE) and first-order differential state equation (SE):

PE: $\varphi=L_{M}(x, i, t) i$,

$\mathrm{SE}: \dot{x}=\frac{d}{d t} x=f_{i}(x, i, t)$.

In general, $L_{M}()$ and $f_{i}()$ are nonlinear functions, which depend on the concrete physical implementation of the current-controlled meminductive system or meminductor. The suffix $i$ denotes the current-controlled system.

Analogously, the FCMLS for modeling the flux-controlled meminductor can be defined as follows:

PE: $i=\Lambda_{M}(x, \varphi, t) \varphi$

$\mathrm{SE}: \dot{x}=\frac{d}{d t} x=f_{\varphi}(x, \varphi, t)$,
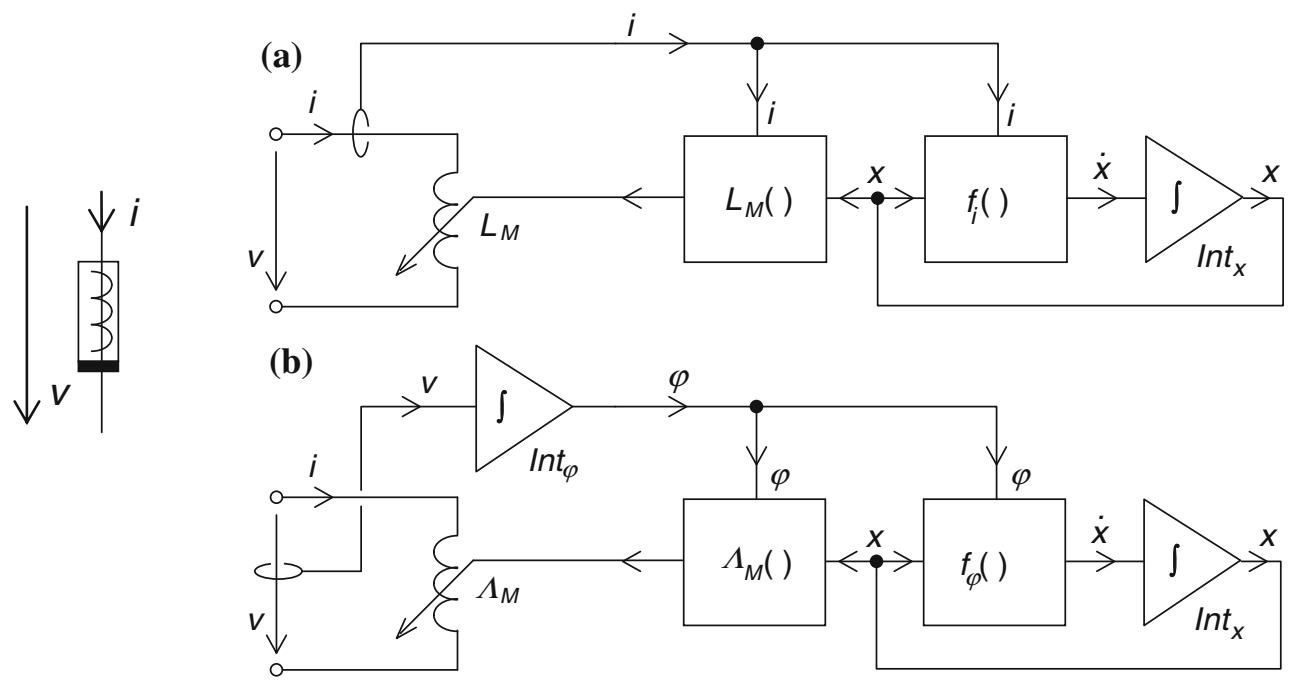

Fig. 1 Schematic symbol of the meminductor and block diagrams of SPICE modeling of a current-controlled, b flux-controlled meminductor 
where $\Lambda_{M}\left(\right.$ ) and $f_{\varphi}($ ) are nonlinear functions. The suffix $\varphi$ denotes the flux-controlled system.

The functions $f_{i}()$ and $f_{\varphi}()$ should also model the so-called boundary effects, which cause the decrease of the speed of state variable to zero if the device state approaches its physical limits [20]. Details will be given in the following section.

Equations 9-12 can serve as a starting point for constructing PSPICE models of current- and flux-controlled meminductors operating on the basis of various physical principles. The basic schematics of such modeling are given in Fig. 1(a) and (b), together with the schematic symbol of the meminductor. The symbol should be used in accordance with [12]: when a positive voltage is applied to the upper terminal with respect to the terminal denoted by the black thick line, the meminductance is increased.

Port equations (9) and (11) are modeled by inductors with inductances controlled through the blocks $L_{M}\left(\right.$ ) and $\Lambda_{M}$ ( ). Input signals of these blocks are the system state $x$ and the meminductor current (Fig. 1a) or flux (Fig. 1b). The flux is computed as a time-domain integral of meminductor voltage (see the block $I n t_{\varphi}$ in Fig. 1b). Differential state equations (10) and (12) are modeled by the integrator $\operatorname{Int}_{x}$ and the nonlinear blocks $f_{i}()$ and $f_{\varphi}()$, respectively.

The varying inductors in Fig. 1 can be modeled in PSPICE as follows. The general equation of the inductor with varying inductance $L_{M}$ or with varying inverse inductance $\Lambda_{M}=1 / L_{M}$

$$
\begin{aligned}
& \varphi(t)=\varphi(0)+\int_{0}^{t} v(\xi) d \xi, \text { or } \\
& L_{M}(t) i(t)=L_{M}(0) i(0)+\int_{0}^{t} v(\xi) d \xi,
\end{aligned}
$$

where $\varphi(0)$ and $i(0)$ are the initial flux and current at time 0 , can be rewritten in the form

$i(t)=\Lambda_{M}(t)\left[L_{M}(0) i(0)+\int_{0}^{t} v(\xi) d \xi\right]$.

This equation can be modeled in PSPICE via a controlled current source. Note that the right-side integral is a flux, which must be computed. The mathematical formula of the inverse meminductance will depend on the concrete physical implementation of the meminductor.

\section{Example of SPICE modeling of meminductor}

Figure 2 shows a simple electro-mechanical model of meminductor. The coil has one fixed (on the left side) and one sliding terminal, which can be moved, depending on the circuit quantities described below, within limits defined by distances $l_{\min }$ and $l_{\max }$ from the fixed terminal. The slider positions $l_{\min }$ and $l_{\max }$ determine the limiting values of the coil inductances $L_{\min }$ and $L_{\max }$. Let us define the dimensionless state variable $x$

$x=\frac{l-l_{\min }}{l_{\max }-l_{\min }} \in(0,1)$.

The coil inductance $L$ is, roughly speaking, proportional to the square of the number of turns $N$ :

$L \approx N^{2} / R_{M}$,

where $R_{M}$ is the magnetic resistance of the magnetic part of the coil.

Then the inductance $L_{M}$ of the meminductor in Fig. 2 depends on the state variable $x$ approximately as follows:

$L(t) \approx\left[\sqrt{L_{\min }}+x(t)\left(\sqrt{L_{\max }}-\sqrt{L_{\min }}\right)\right]^{2}$

Consider the current-controlled meminductor. According to Eq. 10, the time derivative of the state variable, i.e. the speed of the motion of the normalized distance $x$ of the coil slider from the fixed terminal in Fig. 2, must depend on the inductor current. Analogously to the charge-controlled HP memristor in [3] and memcapacitor in [17], consider state equation (10) of the current-controlled meminductor in the form

$\dot{x}=k \cdot i(t) \cdot \operatorname{window}(x)$

The rate of the change of the slider position is directly proportional to the meminductor current and to the mobility factor $k$. The purpose of the nonlinear function window $(x)$ is to model the rate decrease to zero when the slider approaches the limit positions $l_{\min }$ and $l_{\max }$. As known from papers on HP memristor, the modeling of such boundary effects can be accomplished by several types of window functions, which provide a transition to zero values such as the rectangular [12, 21], Joglekar [20], or Biolek [3] window, denoted by $R, J$, and $B$ subscripts in the formulae below:

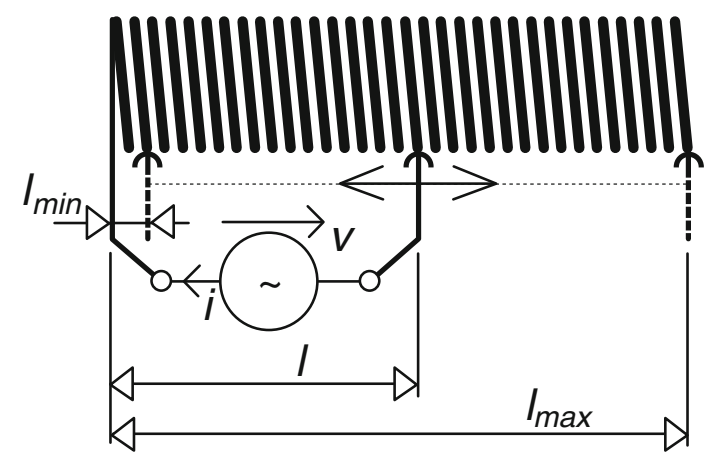

Fig. 2 Electro-mechanical model of the meminductor 
$\operatorname{window}_{R}(x)=\operatorname{stp}(x)-\operatorname{stp}(x-1)$,

window $_{J}(x)=1-(2 x-1)^{2 p}$,

$\operatorname{window}_{B}(x)=1-(x-\operatorname{stp}(-x d))^{2 p}$

Here $\operatorname{stp}(x)$ is a step function, i.e. $\operatorname{stp}(x)=1$ when $x \geq 0$, $\operatorname{stp}(x)=0$ when $x<0, p$ is a positive integer, and $x d$ is a quantity which evaluates the direction of the change of the state variable, i.e. $x d>0$ when $x$ increases, and $x d \leq 0$ when $x$ decreases or is constant.

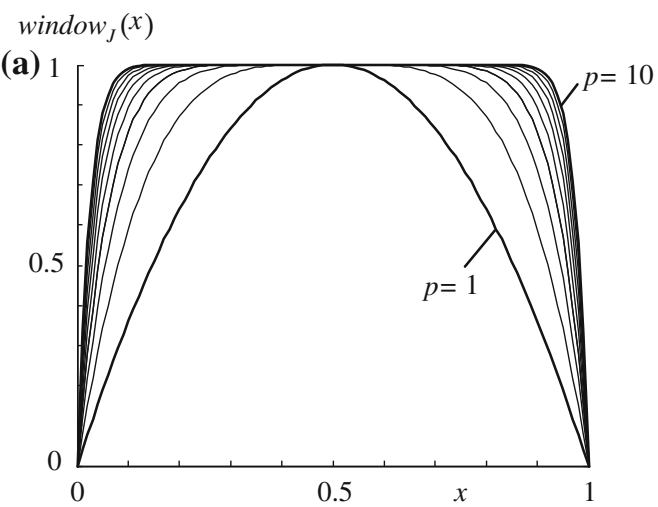

Figure 3(a) shows that if the control parameter $p$ increases, the Joglekar window converges to the rectangular window. The basic problem of this window consists in the fact that when the state variable of the device is in its terminal value 0 or 1 , no external stimulus can change this state because the time derivative of the state variable in (17) is zero, independently of the terminal signals. Such a discrepancy between the behavior of the model and the requirements for the operation of a real circuit element is

window $_{B}(x)$

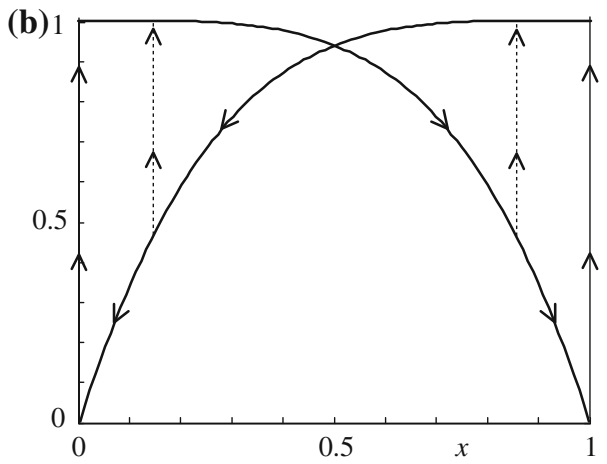

Fig. 3 Window functions according to Joglekar (a) and Biolek (b), the latter for $p=2$ [3]

Table 1 PSPICE subcircuit of the meminductor from Fig. 2

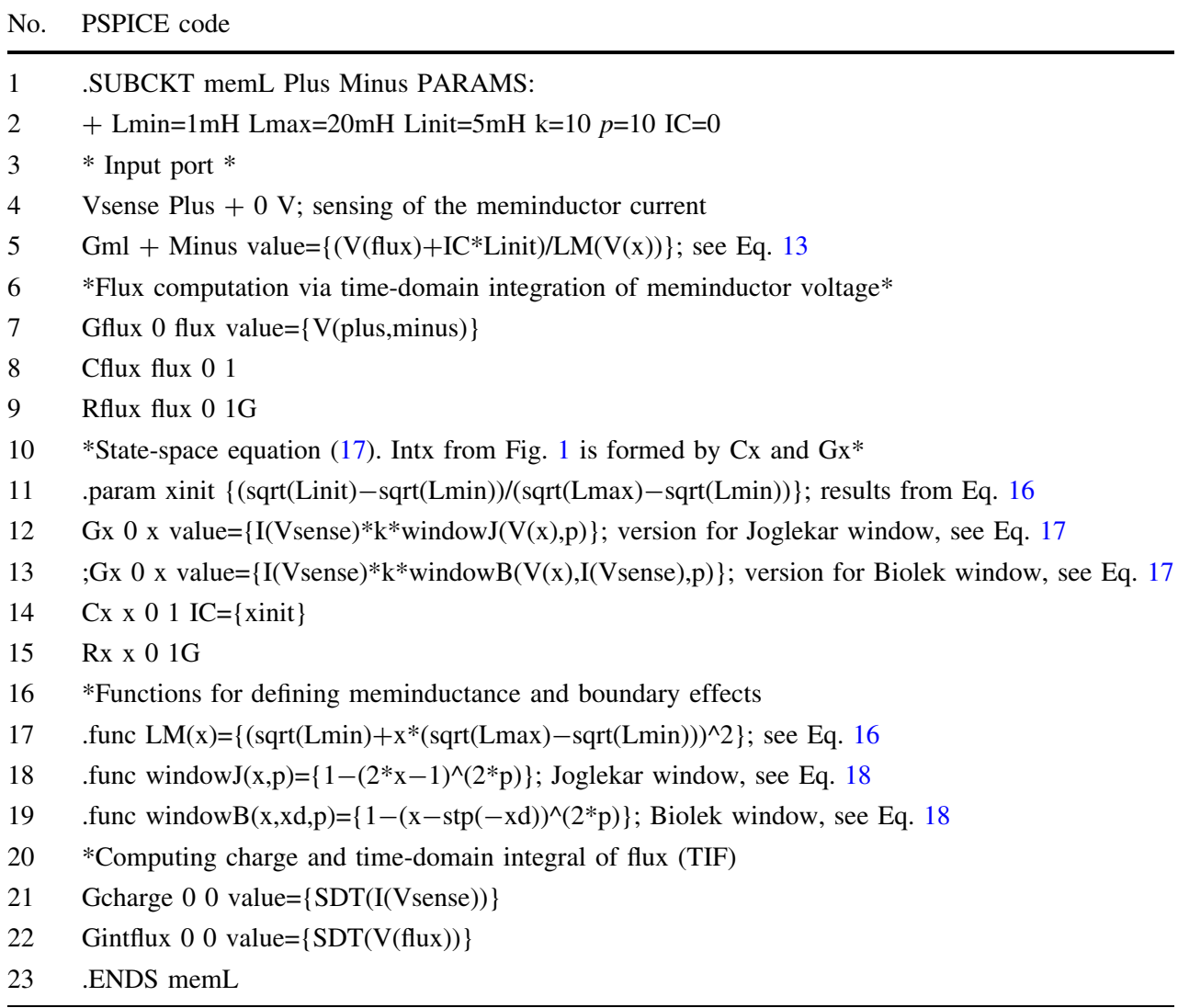


Fig. 4 PSPICE analysis of meminductor from Table 1 excited by harmonic current source. Boundary effects are modeled via Joglekar window
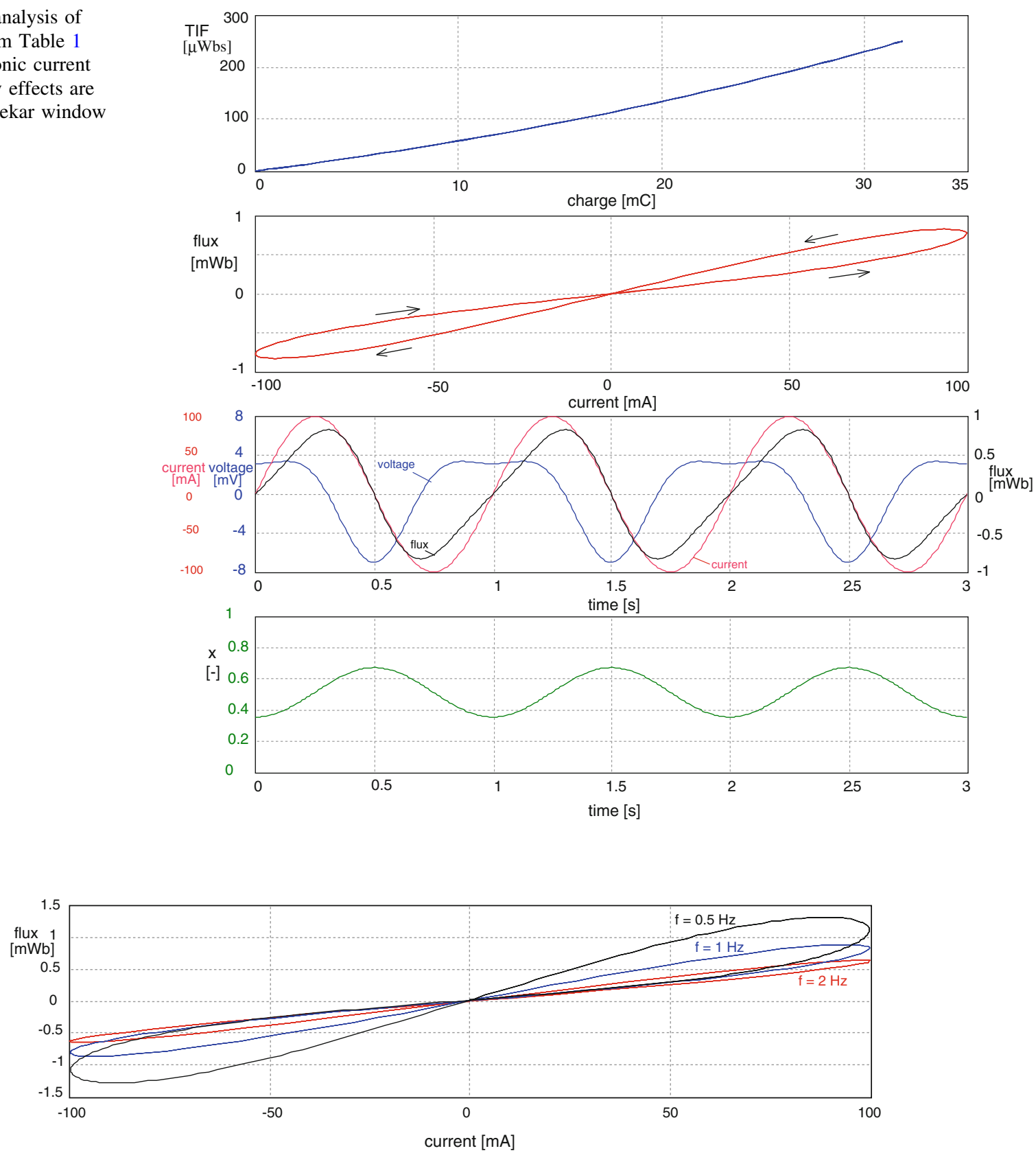

Fig. 5 PSPICE analysis of meminductor from Table 1 excited by harmonic current source. Boundary effects are modeled via Joglekar window. The memory effect decreases with increasing frequency of excited signal

resolved by the Biolek window, which reflects the fact that the speeds of receding from and approaching the limit positions of the slider are different. An illustration of how this window works is given in Fig. 3(b) for the control parameter $p=2$. Details can be found in [3,9]. As a conclusion, the more sophisticated Biolek window models well the device behavior also near its boundary states, but numerical problems in transient analysis due to the discontinuities at boundary points can appear more frequently than in the case of the Joglekar window. The Joglekar window can be advantageously used for cases when the mem-element operates far enough from its boundary states. Note that when applying the Biolek window, constitutive relation (1) will now depend on the way the meminductor interacts with the surrounding networks. However, it is only a consequence of the fact that the device in Fig. 2 is not an ideal meminductor but a more general meminductive system. 
Fig. 6 PSPICE analysis of meminductors excited by trapezoidal pulse current sources, with boundary effects modeled by Joglekar and Biolek windows. Figures (a) and (b) show initial transients whereas figures (c) and (d) display the steady-state behavior. The meminductor parameters are $L_{\text {min }}=100 \mu \mathrm{H}$, $L_{\text {max }}=2 \mathrm{mH}, L_{\text {init }}=1 \mathrm{mH}$, $k=10 \mathrm{~A}^{-1} \mathrm{~s}^{-1}, p=10$
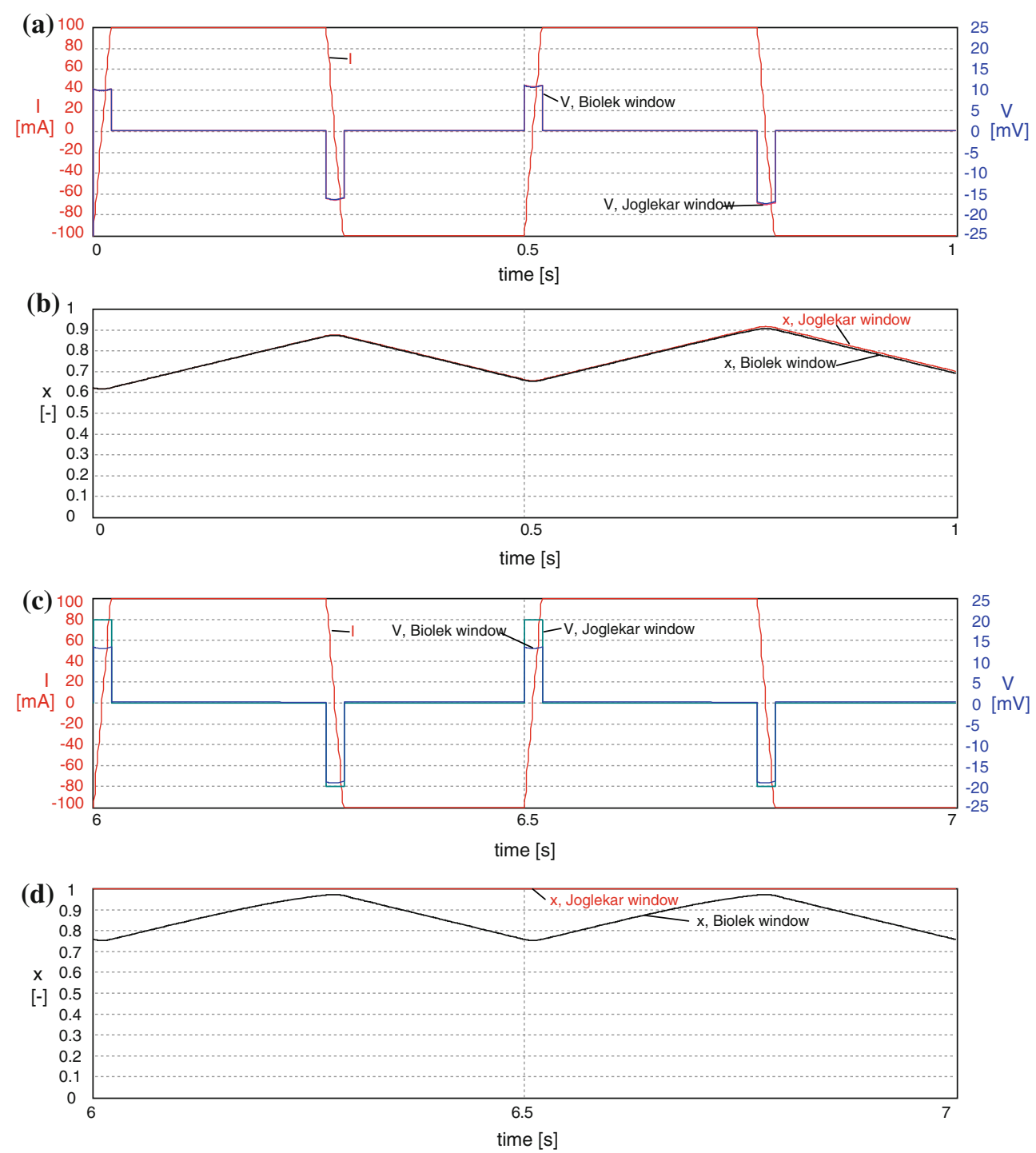

The SPICE subcircuit, modeling the meminductor from Fig. 2, is shown in Table 1 together with the self-explanatory notes.

The Vsense voltage source, defined on line 4 , serves to sense the meminductor current. The value of this current is needed for modeling the state equation on line 12 or 13 as well as for computing the charge on line 21 . The code on line 12 can be replaced by the text on line 13 when the Biolek window is used instead of the Joglekar window. The integrator $I n t_{x}$ in Fig. 1(a) is accomplished by a capacitor $C_{x}$ with $1 \mathrm{~F}$ capacitance, charged from a current source $G_{x}$, which provides current according to Eq. 17. A shunting resistor $R_{x}$ is needed in order to provide the DC path to the ground. The initial state $x_{i n i t}$ of the device is derived from the initial value of inductance $L_{i n i t}$, see line 11 . The flux computation as a time-domain integral of meminductor voltage is done similarly on lines 7-9.
In order to easily visualize constitutive relation (1), the charge and TIF computations are performed on lines 21 and 22. For simplicity, the time-domain integrations are provided via a PSPICE function SDT, not by means of the current source and capacitor. To save model nodes, the computation is arranged via current sources with each terminal grounded. The values of these currents are equal to the charge and TIF and thus they can be used for the visualization of the CR. Note that the SDT function was not utilized in the above-mentioned models of integrators. The main reason for not using the SDT function is that the precision of these integrators can then be adjusted via a proper selection of the component parameters.

The results of PSPICE transient analysis are shown in Fig. 4. The analysis was performed for the meminductor with default parameters given in Table 1 and the Joglekar window, excited by a harmonic current source with an 
Fig. 7 Steady-state characteristics of the meminductors analyzed in Fig. 6: flux-current characteristics for Joglekar (a) and Biolek (b) windows, TIF-charge characteristics for Joglekar (c) and Biolek (d) windows
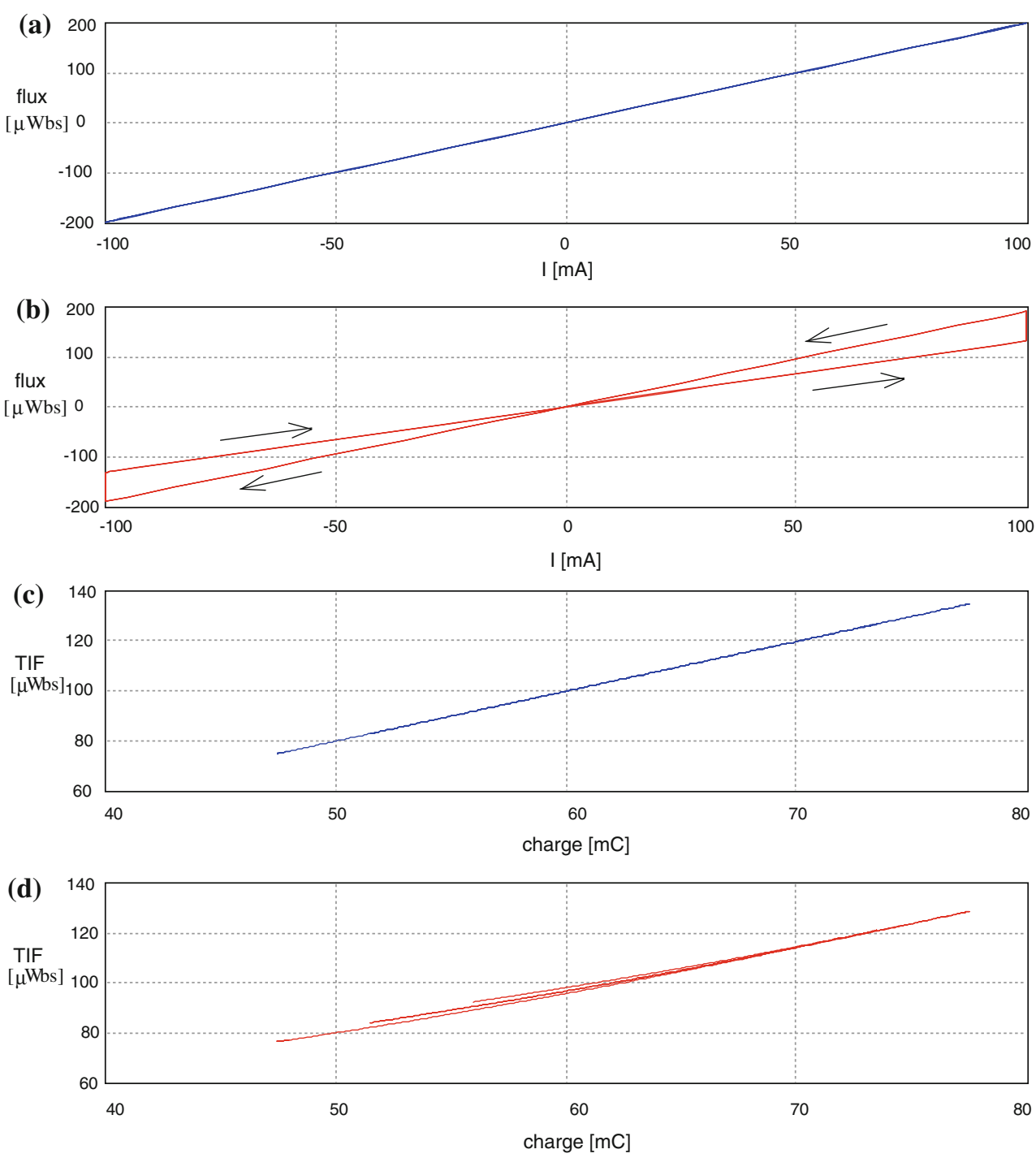

amplitude of $100 \mathrm{~mA}$ and a frequency of $1 \mathrm{~Hz}$, with an auxiliary $1 \mathrm{G} \Omega$ shunting resistor. The results shown in Fig. 4 clearly demonstrate three basic fingerprints of the meminductor [11]: Unambiguous constitutive relation (1) (Fig. 4a), the pinched hysteretic loop in the flux-current characteristic (Fig. 4b), and the identical time instants when the flux and current waveforms cross the zero levels (Fig. 4c). Figure 4(d) shows the variation of the state variable $x$ due to changing the terminal current and voltage.

Figure 5 demonstrates another well-known fact from the world of mem-devices: if the frequency of the signal excitation increases, the hysteretic effect in the flux-current characteristic is gradually suppressed.

Figure 6 offers a comparison of meminductor behaviors when the boundary effects are modeled by the Joglekar and the Biolek windows. Meminductors are excited by identical current sources with symmetrical periodical trapezoidal pulse waveforms with MIN/MAX values of $-100 \mu \mathrm{A} /$ $+100 \mu \mathrm{A}$, with a repeating frequency of $2 \mathrm{~Hz}$, and with $5 \mathrm{~ms}$ rise and fall times. Figure 6(a) and (b) show the initial transients whereas Fig. 6(c) and (d) display the steady-state meminductor voltages, currents, and state variables $x$. Note that whereas the initial transients are almost identical (see Fig. 6a, b), in the steady-state the meminductor modeled by the Joglekar window operates in the regime of $x=1$, due to the effect of locking in on the boundary state in which the time derivative of the state variable is zero, and thus it behaves as a conventional linear inductor. The corresponding flux-current characteristic in Fig. 7(a) is a line without any hysteretic behavior. By contrast, the operation of the meminductor with the Biolek window exhibits a steady-state variation of the state variable $x$ (see Fig. 6d), and the hysteretic loop in the flux-current characteristic is evident in Fig. 7(b). Note that the constitutive TIF-charge relation in Fig. $7(\mathrm{~d})$ is not ambiguous because the 
meminductor now behaves as a more complicated meminductive system.

\section{Conclusions}

The methodology described here facilitates effective modeling of meminductive systems of various physical natures in the SPICE-family programs for circuit simulation. An example of the modeling of a current-controlled meminductor whose inductance is changed depending on a state variable, controlled by the meminductor current, is analyzed in detail. The SPICE modeling of flux-controlled meminductive systems can be figured out analogously, following the general model in Fig. 1(b).

Acknowledgments Research described in the paper was supported by the Czech Science Foundation under grant No. P102/10/1614, and by the research programmes of BUT Nos MSM0021630503/13 and UD Brno No. MO FVT0000403, Czech Republic.

Open Access This article is distributed under the terms of the Creative Commons Attribution Noncommercial License which permits any noncommercial use, distribution, and reproduction in any medium, provided the original author(s) and source are credited.

\section{References}

1. Strukov, D. B., Snider, G. S., Stewart, D. R., \& Williams, R. S. (2008). The missing memristor found. Nature, 453, 80-83.

2. Benderli, S., \& Wey, T. A. (2009). On SPICE macromodelling of $\mathrm{TiO}_{2}$ memristors. Electronics Letters, 45(7), 377-379.

3. Biolek, Z., Biolek, D., \& Biolková, V. (2009). SPICE model of memristor with nonlinear dopant drift. Radioengineering, 18(2), 210-214.

4. Shin, S., \& Kang, S.-M. (2010). Compact models for memristors based on charge-flux constitutive relationships. IEEE Transactions on Computer-Aided Design of Integrated Circuits and Systems, 29(4), 590-598.

5. Rák, A., \& Cserey, G. (2010). Macromodeling of the memristor in SPICE. IEEE Transactions on Computer-Aided Design of Integrated Circuits and Systems, 29(4), 632-636.

6. Batas, D., \& Fiedler, H. (2010). A memristor Spice implementation and a new approach for magnetic flux controlled memristor modeling. IEEE Transactions on Nanotechnology. doi:10.1109/ TNANO.2009.2038051.

7. Chen, Y., \& Wang, X. (2009). Compact modeling and corner analysis of spintronic memristor. In 2009 IEEE/ACM International Symposium on Nanoscale Architectures, pp. 7-12.

8. Zhang, Y., Zhang, X., \& Yu, J. (2009). Approximated SPICE model for memristor. In International conference on communications, circuits and systems, ICCCAS, pp. 928-931.

9. Kavehei, O., Iqbal, A., Kim, Y. S., Eshraghian, K., Al-Sarawi, S. F., \& Abbot, D. (2010). The fourth element: Characteristics, modelling, and electromagnetic theory of the memristor. arXiv:1002.3210v1 [cond-mat.mes-hall] 17 Feb 2010.

10. Chua, L. O. (1971). Memristor-The missing circuit element. IEEE Transactions on Circuit Theory, CT-18(5), 507-519.

11. Chua, L. O. The introductory talk. In Memristor and memristive systems symposium, University of California, Berkeley, 2008.

12. Di Ventra, M., Pershin, Y. V., \& Chua, L. O. (2009). Circuit elements with memory: Memristors, memcapacitors and meminductors. Proceedings of the IEEE, 97(10), 1717-1724.

13. Martinez, J., Di Ventra, M., \& Pershin, Y. V. (2009). Solid-state memcapacitor. arXiv:0912.4921v2 [cond-mat.mes-hall] $31 \mathrm{Dec}$ 2009.

14. Krems, M., Pershin, Y.V., \& Di Ventra, M. (2010). Ionic memcapacitive effects in nanopores. arXiv:1001.0796v1 [cond-mat.soft] 6 Jan 2010.

15. Di Ventra, M., Pershin, Y. V., \& Chua, L. O. (2009). Putting memory into circuit elements: Memristors, memcapacitors, and meminductors. Proceedings of the IEEE, 97(8), 1371-1372.

16. Biolek, D., Biolek, Z., \& Biolková, V. (2009). SPICE modeling of memristive, memcapacitative and meminductive systems. In Proc. int. conf. ECCTD '09, Antalya, Turkey, pp. 249-252.

17. Biolek, D., Biolek, Z., \& Biolková, V. (2010). SPICE modelling of memcapacitor. Electronics Letters, 46(7), 520-522.

18. Chua, L. O. (2003). Nonlinear circuit foundations for nanodevices. Part I. The four-Element Torus. Proceedings of the IEEE, 91(11), 1830-1859.

19. Chua, L. O., \& Kang, S. M. (1976). Memristive devices and systems. Proceedings of the IEEE, 64(2), 209-223.

20. Joglekar, Y. N., \& Wolf, S. J. (2009). The elusive memristor: Properties of basic electrical circuits. European Journal of Physics, 30, 661-675.

21. Pershin, Y. V., \& Di Ventra, M. (2010). Memristive circuits simulate memcapacitors and meminductors. Electronics Letters, 46(7), 517-518.

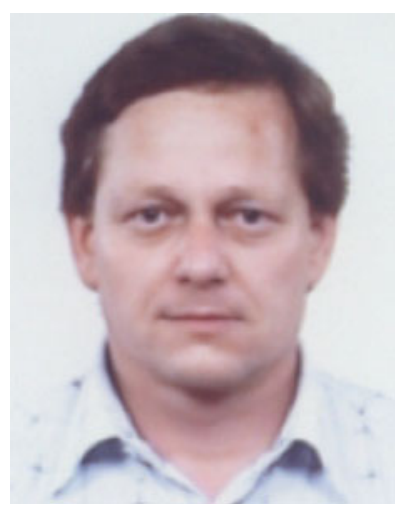

Dalibor Biolek He received the M.Sc. degree in Electrical Engineering from the Brno University of Technology, Czech Republic, in 1983, and the Ph.D. degree in Electronics from the Military Academy Brno, Czech Republic, in 1989, focusing in algorit of the symbolic and numerical computer analysis of electronic circuits with a view to the linear continuous-time and switched filters. He is currently with the Department of EE, University of Defence Brno (UDB), and with the Department of Microelectronics, Brno University of Technology (BUT), Czech Republic. His scientific activity is directed to the areas of general circuit theory, frequency filters, and computer simulation of electronic systems. He has published over 250 papers and is author of a book on circuit analysis and simulation. At present, he is professor at the BUT and UDB in the field of Theoretical Electrical Engineering. $\mathrm{He}$ is a member of the CAS/COM Czech National Group of IEEE. He is also the president of Commission C of the URSI National Committee for the Czech Republic. 


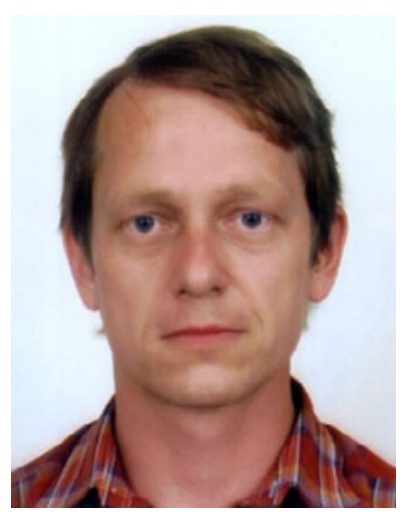

Zdeněk Biolek He received the M.Sc. degree in 1983, and the Ph.D. degree in 2001 from Brno University of Technology, Czech Republic, both in Electrical Engineering with a view to thermodynamics. Until 1992 he was the principal research worker at Czech Semiconductor Company TESLA Rožnov. He is currently with the SŠIER Rožnov p.R., Czech Republic. $\mathrm{He}$ has authored several special electronic instruments for manufacturing and testing integrated circuits. He is also the author and co-author of papers from the area of the utilization of variational principles in circuit theory and the stability testing of resistive circuits.

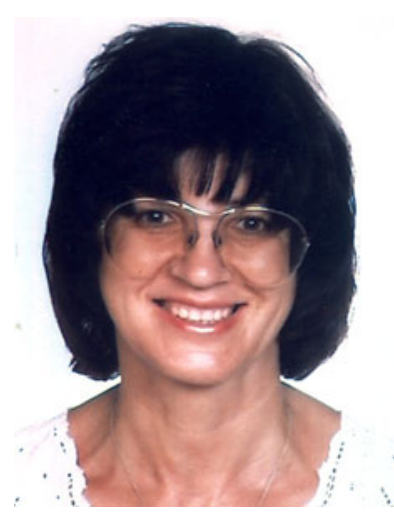

Viera Biolkova He received her M.Sc. degree in Electrical Engineering from the Brno University of Technology, Czech Republic, in 1983. She joined the Department of Radio Electronics in 1985 , and is currently working as a Research Assistant at the Department of Radio Electronics, Brno University of Technology (BUT), Czech Republic. Her research and educational interests include signal theory, analog signal processing, and digital electronics. 